\title{
Multidromion Soliton and Rouge Wave for the $(2+1)$-Dimensional Broer-Kaup System with Variable Coefficients
}

\author{
Zitian Li \\ College of Mathematics and Information Science, Qujing Normal University, Qujing, Yunnan 655011, China \\ Correspondence should be addressed to Zitian Li; lizitian88@163.com
}

Received 26 November 2014; Revised 6 March 2015; Accepted 9 March 2015

Academic Editor: Kishin Sadarangani

Copyright (C) 2015 Zitian Li. This is an open access article distributed under the Creative Commons Attribution License, which permits unrestricted use, distribution, and reproduction in any medium, provided the original work is properly cited.

Broad new families of rational form variable separation solutions with two arbitrary lower-dimensional functions of the $(2+1)$ dimensional Broer-Kaup system with variable coefficients are derived by means of an improved mapping approach and a variable separation hypothesis. Based on the derived variable separation excitation, some new special types of localized solutions such as rouge wave, multidromion soliton, and soliton vanish phenomenon are revealed by selecting appropriate functions of the general variable separation solution.

\section{Introduction}

Searching for explicit solutions of nonlinear evolution equations by using various different methods is useful and meaningful in physical science and nonlinear science. Many powerful methods have been presented, such as inverse scattering transform [1], Hirota's bilinear form method [2], two-soliton method [3], homoclinic test technique [4-6], the Bäcklund transformation method [7], three-wave type of ansätz approach $[8,9]$, projective equation method [10], and multilinear variable separation method [11].

In the line with the development of symbolic computation, much work has been focused on the various extensions and application of the known algebraic methods to construct solutions of nonlinear evolution equations [12-14].

In this paper, we will apply a projective equation method [15] with a variable separation hypothesis to look for new families of variable separation solutions to the $(2+1)$ dimensional Broer-Kaup system with variable coefficients $(\mathrm{vcBK})[16]$ :

$$
\begin{gathered}
u_{y t}-B(t)\left[u_{x x y}-2\left(u u_{x}\right)_{y}-2 v_{x x}\right]=0, \\
v_{t}+B(t)\left[v_{x x}+2(u v)_{x}\right]=0,
\end{gathered}
$$

where $B(t)$ is an arbitrary function of time $t$ and $B(t) \neq 0$. When $B(t)=1$, the system reduces to the celebrated $(2+1)$ dimensional Broer-Kaup system.

The vcBK system (1) is an important mathematical model in nonlinear optics, plasma physics, and statistical physics [16-18]. In our work, we apply a projective equation method and a variable separation hypothesis to the BK equations (1) and obtain its exact excitations. We give some selected oscillating solitons, multidromion soliton, and crosslike fractal structures by selecting appropriate functions in the general variable separation solution of the $\mathrm{vcBK}$ equations to demonstrate some interesting outcomes most of which are new by comparing with the solutions of the references.

\section{The Nontraveling Wave Solutions of the vcBK Equations}

By letting $v=u_{y}$, (1) can be converted into a new equation

$$
u_{y t}+B(t)\left[u_{x x y}+2\left(u u_{x}\right)_{y}\right]=0 .
$$


Suppose that the solution of (2) can be expressed as follows:

$$
u=a_{0}(X)+\sum_{i=1}^{n} \frac{a_{i}(X)}{F^{i}(\phi(X))}+\sum_{i=1}^{n} \frac{b_{i}(X) F^{i}(\phi(X))}{(1+r(x, t) F(\phi(X)))^{i}}
$$

where $a_{0}(X), a_{i}(X), b_{i}(X)(i=1,2, \ldots, n)$, and $\phi(X)$ are all functions of $X$ to be determined, $r(x, t)$ is arbitrary function of $\{x, t\}$, and $F(\phi(X))$ satisfies the Riccati equation

$$
F^{\prime}(\phi(X))=\delta+F^{2}(\phi(X))
$$

with $\delta$ an arbitrary constant. Notice that the Riccati equation (4) possesses the following solutions:

(i) when $\delta=0, F(\phi(X))=-1 / \phi(X)$;

(ii) when $\delta<0, F(\phi(X))=-\sqrt{-\delta} \tanh (\sqrt{-\delta} \phi(X))$;

(iii) when $\delta>0, F(\phi(X))=\sqrt{\delta} \tan (\sqrt{\delta} \phi(X))$.

By balancing the linear term of the highest order with the nonlinear term in (2), we get $n=1$; then according to the ansätz (3), the solution of (2) reads

$$
\begin{aligned}
u(x, y, t)= & a_{0}(x, y, t) \\
& +\frac{a_{1}(x, y, t)}{F(\phi(x, y, t))}+\frac{a_{2}(x, y, t) F(\phi(x, y, t))}{1+r(x, t) F(\phi(x, y, t))},
\end{aligned}
$$

where $a_{0}(x, y, t), a_{1}(x, y, t) a_{2}(x, y, t)$, and $\phi(x, y, t)$ are arbitrary functions of $(x, y, t)$ to be determined, $r(x, t)$ is arbitrary functions of $\{x, t\}$ to be determined, and $F$ satisfies (4). Inserting (5) with (4) into (2), selecting the variable separation ansätz

$$
\phi=p(x, t)+q(y)
$$

with $r(x, t)=0$ (for simplicity), and eliminating all the coefficients of polynomials of $F$, one gets a set of partial differential equations as follows:

$$
6 B \delta^{2} a_{1}^{2} p_{x} q_{y}-6 B \delta^{3} a_{1} p_{x}^{2} q_{y}=0
$$

$$
\begin{aligned}
& 6 B a_{2}^{2} p_{x} q_{y}+6 B a_{2} p_{x}^{2} q_{y}=0 \\
& 2 \delta^{2} a_{1} p_{t} q_{y}+4 B \delta^{2} a_{0} a_{1} p_{x} q_{y}+2 B \delta^{2} a_{1} p_{x x} q_{y} \\
& -4 B \delta a_{1} a_{1 y} p_{x}+2 B \delta^{2} a_{1 y} p_{x}^{2}-4 B \delta a_{1} a_{1 x} q_{y} \\
& +4 B \delta^{2} a_{1 x} p_{x} q_{y}=0, \\
& 2 a_{2} p_{t} q_{y}+4 B a_{0} a_{2} p_{x} q_{y}+2 B a_{2} p_{x x} q_{y} \\
& +4 B a_{2} a_{2 y} p_{x}+2 B a_{2 y} p_{x}^{2}+4 B a_{2} a_{2 x} q_{y} \\
& +4 B a_{2 x} p_{x} q_{y}=0 \text {, } \\
& 8 B \delta a_{1}^{2} p_{x} q_{y}-8 B \delta^{2} a_{1} p_{x}^{2} q_{y}-\delta a_{1 t} q_{y}-2 B \delta a_{1} a_{0 y} p_{x} \\
& -\delta a_{1 y} p_{t}-2 B \delta a_{0} a_{1 y} p_{x}-B \delta a_{1 y} p_{x x}-2 B \delta a_{1} a_{0 x} q_{y} \\
& -2 B \delta a_{0} a_{1 x} q_{y}+2 B a_{1 x} a_{1 y}+2 B a_{1} a_{1 x y} \\
& -2 B \delta a_{1 x y} p_{x}-B \delta a_{1 x x} q_{y}=0, \\
& 8 B \delta a_{2}^{2} p_{x} q_{y}+8 B \delta a_{2} p_{x}^{2} q_{y}+a_{2 t} q_{y}+2 B a_{2} a_{0 y} p_{x} \\
& +a_{2 y} p_{t}+2 B a_{0} a_{2 y} p_{x}+B a_{2 y} p_{x x}+2 B a_{2} a_{0 x} q_{y} \\
& +2 B a_{0} a_{2 x} q_{y}+2 B a_{2 x} a_{2 y}+2 B a_{2} a_{2 x y}+2 B a_{2 x y} p_{x} \\
& +B a_{2 x x} q_{y}=0 \\
& 2 \delta a_{1} p_{t} q_{y}+4 B \delta a_{0} a_{1} p_{x} q_{y}+2 B \delta a_{1} p_{x x} q_{y} \\
& -4 B a_{1} a_{1 y} p_{x}+2 B \delta a_{1 y} p_{x}^{2}+a_{1 y t}+2 B a_{0 x} a_{1 y} \\
& -4 B a_{1} a_{1 x} q_{y}+4 B \delta a_{1 x} p_{x} q_{y}+2 B a_{0 y} a_{1 x} \\
& +2 B a_{0 x y} a_{1}+2 B a_{0} a_{1 x y}+B a_{1 x x y}=0 \text {, } \\
& 2 \delta a_{2} p_{t} q_{y}+4 B \delta a_{0} a_{2} p_{x} q_{y}+2 B \delta a_{2} p_{x x} q_{y} \\
& +4 B \delta a_{2} a_{2 y} p_{x}+2 B \delta a_{2 y} p_{x}^{2}+a_{2 y t}+2 B a_{0 x} a_{2 y} \\
& +4 B \delta a_{2} a_{2 x} q_{y}+4 B \delta a_{2 x} p_{x} q_{y}+2 B a_{0 y} a_{2 x} \\
& +2 B a_{0 x y} a_{2}+2 B a_{0} a_{2 x y}+B a_{2 x x y}=0 \text {, } \\
& 2 B a_{1}^{2} p_{x} q_{y}+2 B \delta^{2} a_{2}^{2} p_{x} q_{y}-2 B \delta a_{1} p_{x}^{2} q_{y} \\
& +2 B \delta^{2} a_{2} p_{x}^{2} q_{y}-a_{1 t} q_{y}+\delta a_{2 t} q_{y}-2 B a_{1} a_{0 y} p_{x} \\
& +2 B \delta a_{2} a_{0 y} p_{x}-a_{1 y} p_{t}-2 B a_{0} a_{1 y} p_{x}-B a_{1 y} p_{x x} \\
& +\delta a_{2 y} p_{t}+2 B \delta a_{0} a_{2 y} p_{x}+B \delta a_{2 y} p_{x x}+a_{0 y t} \\
& -2 B a_{0 x} a_{1} q_{y}+2 B \delta a_{0} a_{2 x} q_{y}+2 B a_{1 y} a_{2 x} \\
& +2 B a_{0} a_{0 x y}+2 B a_{1 x y} a_{2}-2 B a_{1 x y} p_{x}+2 B a_{1} a_{2 x y} \\
& +2 B \delta a_{2 x y} p_{x}-B a_{1 x x} q_{y}+B \delta a_{2 x x} q_{y}+B a_{0 x x y}=0 \text {. }
\end{aligned}
$$


Solving the set of differential equations simultaneously, we obtain the following results.

Case 1. Consider

$$
a_{0}=-\frac{p_{t}+B(t) p_{x x}}{2 B(t) p_{x}}, \quad a_{1}=\delta p_{x}, \quad a_{2}=0, \quad r=0 .
$$

Case 2. Consider

$$
a_{0}=-\frac{p_{t}+B(t) p_{x x}}{2 B(t) p_{x}}, \quad a_{1}=0, \quad a_{2}=-p_{x}, \quad r=0 .
$$

Case 3. Consider

$$
\begin{aligned}
& a_{0}=-\frac{p_{t}+B(t) p_{x x}}{2 B(t) p_{x}}, \quad a_{1}=\delta p_{x}, \\
& a_{2}=-p_{x}, \quad r=0,
\end{aligned}
$$

where $p=p(x, t) q=q(y)$ and $r=r(x, t)$ are three arbitrary functions of $\{x, t\},\{y\},\{x, t\}$, respectively, and $\delta$ is an arbitrary constant.

Next we only list some new rational variable separation solutions by using Case 3; however, Case 2 is the results in $[19,20]$; we omit them for simplification. Therefore, we can obtain exact solutions for (1) as follows.

(I) When $\delta<0$, we can derive the following solitary wave solution:

$$
\begin{aligned}
& u_{1}(x, y, t) \\
& =-\frac{p_{t}+B(t) p_{x x}}{2 B(t) p_{x}} \\
& \quad+\frac{\sqrt{-\delta} p_{x}\left(1+\tanh ^{2}[\sqrt{-\delta}(p(x, t)+q(y))]\right)}{\tanh [\sqrt{-\delta}(p(x, t)+q(y))]}, \\
& v_{1}(x, y, t)=4 \delta p_{x} q_{y} \operatorname{csch}^{2}[2 \sqrt{-\delta}(p(x, t)+q(y))],
\end{aligned}
$$

with two arbitrary functions being $p(x, t)$ and $q(y)$.

(II) When $\delta>0$, we can obtain the following periodic wave solution:

$$
\begin{aligned}
u_{2}(x, y, t)= & -\frac{p_{t}+B(t) p_{x x}}{2 B(t) p_{x}} \\
& +\frac{\sqrt{\delta} p_{x}\left(1-\tan ^{2}[\sqrt{\delta}(p(x, t)+q(y))]\right)}{\tan [\sqrt{\delta}(p(x, t)+q(y))]}, \\
v_{2}(x, y, t) & =-4 \delta p_{x} q_{y} \csc ^{2}[2 \sqrt{\delta}(p(x, t)+q(y))]
\end{aligned}
$$

with two arbitrary functions being $p(x, t), q(y)$.
(III) When $\delta=0$, then we can derive the following variable separation solution:

$$
\begin{gathered}
u_{3}(x, y, t)=-\frac{p_{t}+B(t) p_{x x}}{2 B(t) p_{x}}-\frac{p_{x}}{p(x, t)+q(y)}, \\
v_{3}(x, y, t)=-\frac{p_{x} q_{y}}{(p+q)^{2}}
\end{gathered}
$$

with two arbitrary functions being $p(x, t)$ and $q(y)$.

By using Mathematica, we verify that all the solutions listed above satisfy the original equation (2).

\section{New Localized Excitations}

Owing to the arbitrary functions $p(x, t)$ and $q(y)$ involved in the solutions (11)-(14), it is convenient to excite soliton structure. After some calculations, we construct a new class of structures, such as the multidromion soliton, rouge wave, and the cross-like fractal solitons. Here, we take the solution (12) of the vcBK equations (1) as an example to study the soliton structure excitation.

Case 1. When $p(x, t)$ and $q(y)$ possess the following forms:

$$
\begin{gathered}
p(x, t)=1+0.25 \operatorname{sech}(0.5 x+t) \\
+0.1 \operatorname{sech}(0.2 x+2 t), \\
q(y)=1+\operatorname{sech}(4+y)+0.5 \operatorname{sech}(4-y),
\end{gathered}
$$

respectively, we can obtain the multidromion soliton structures of the $(2+1)$-dimensional $\mathrm{vcBK}$ equation. Figure $1(\mathrm{a})$ clearly indicates that the multidromion moves backwards and forwards over the same path in $x-y$ plane, oscillating in $x$ direction.

Case 2. If $p(x, t)$ and $q(y)$ are taken as

$$
p(x, t)=\frac{1}{4}(x+t)^{2}, \quad q(y)=\frac{1}{8}+\frac{1}{2} y^{2},
$$

respectively, then we obtain a peakon-type rouge wave structure which is exhibited in Figure 1(b).

Case 3. When $p(x, t)$ and $q(y)$ are choosing as

$$
p(x, t)=1+\tanh (x+t), \quad q(y)=1-\tanh (y),
$$

respectively, the physical quantity $v$ shows the soliton vanish phenomenon. Figure 2(a) shows that when $T=x+t<0$, there is a soliton, and as $T=x+t$ increases over 0 , it vanishes very soon.

Case 4. When $p(x, t)$ and $q(y)$ possess the following forms:

$$
\begin{aligned}
& p(x, t)=\exp \left(-\frac{1}{4} x^{2}+2 t-0.5\right), \\
& q(y)=\exp \left(-\frac{1}{2} y^{2}\right)
\end{aligned}
$$

respectively, we have oscillating soliton-type structure. Their plots are presented in Figure 2(b). 


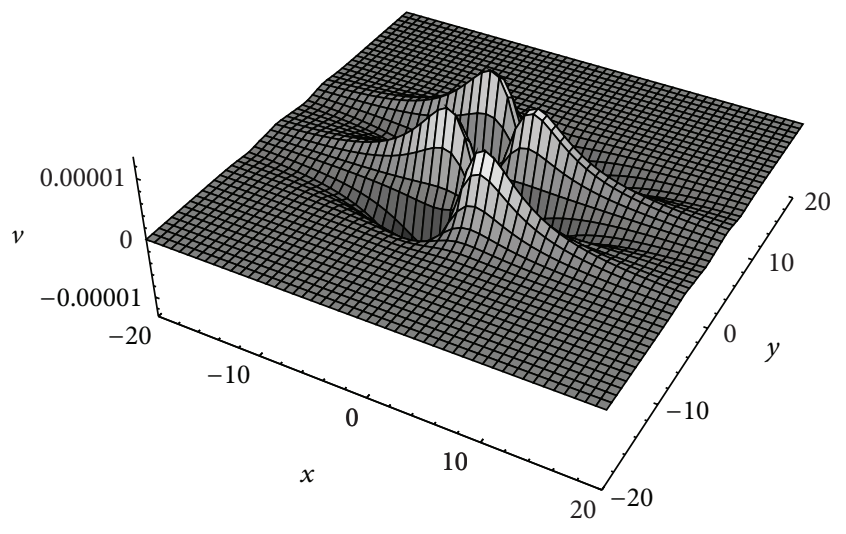

(a)

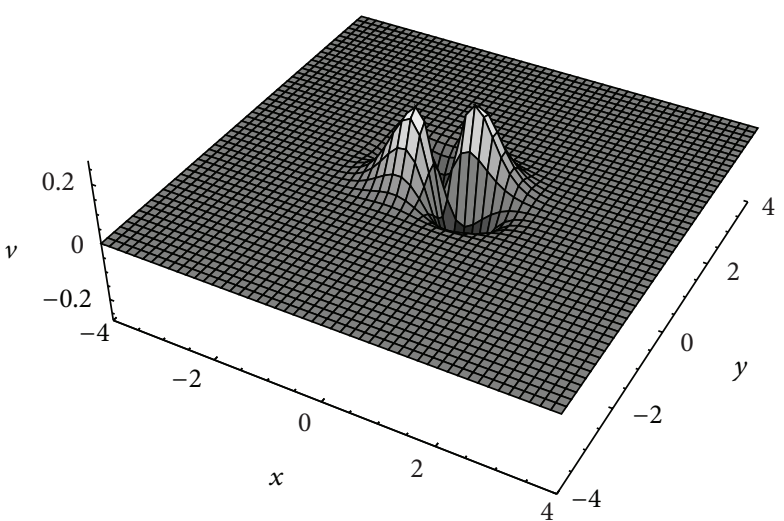

(b)

Figure 1: (a) Multidromion soliton $(\delta=-1, t=0)$. (b) Rouge wave $(\delta=-4, t=0)$.

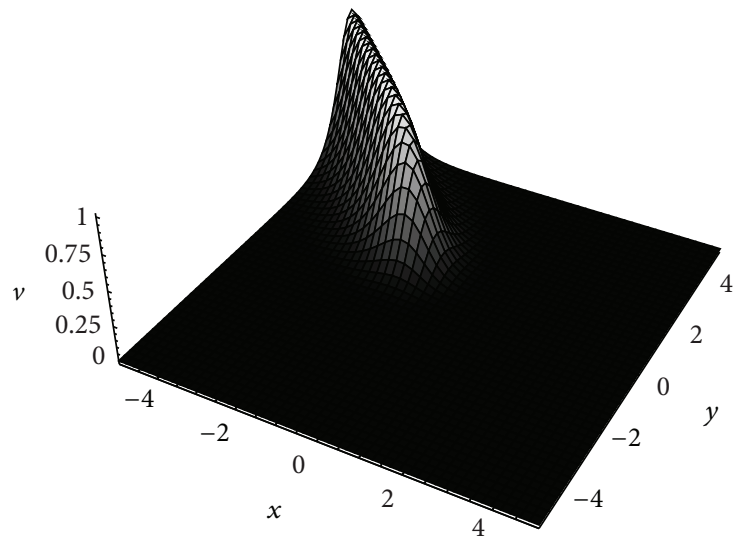

(a)

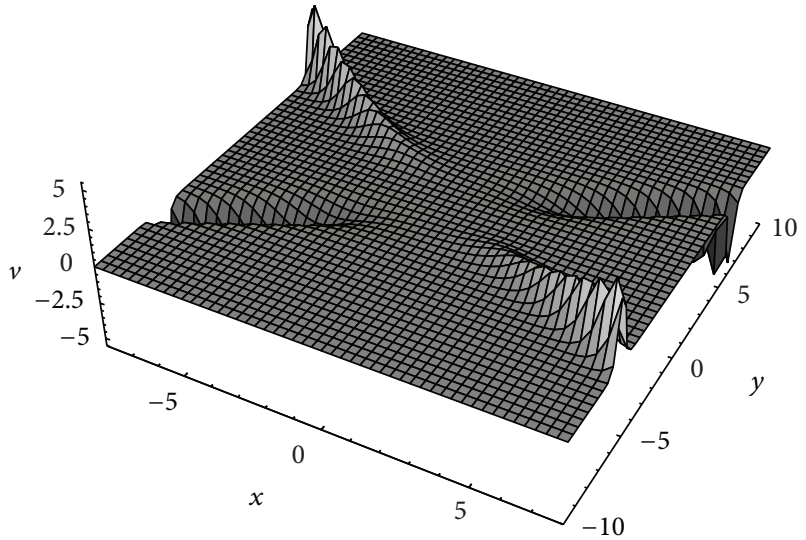

(b)

Figure 2: (a) Soliton vanish phenomenon $(\delta=-1, t=0)$. (b) Oscillating soliton-type structure $(\delta=-1, t=0)$.

Case 5. If $p(x, t)$ and $q(y)$ are selecting as

$$
\begin{aligned}
& p(x, t)=4 \exp [(2 x+t) \cos (x+t)-10], \\
& q(y)=2 \exp [y-10]
\end{aligned}
$$

respectively, we obtain a dromion of periodic oscillation structure which is shown in Figure 3(a).

Case 6. When $p(x, t)$ and $q(y)$ possess the following forms:

$$
p(x, t)=\operatorname{sech}\left[2(x+t)^{2}\right], \quad q(y)=\exp (-2 y),
$$

respectively, we obtain the parabola-type kink solitary wave structure which is shown in Figure 3(b).

Case 7. When $p(x, t)$ and $q(y)$ possess the following forms:

$$
p(x, t)=-2 x \ln \left(x^{4}+t^{2}\right), \quad q(y)=4-y \ln \left(y^{4}\right),
$$

respectively, we obtain the cross-like fractal structures which we can see reported in [21].
Figures 4(a) and 4(b) give the figures of the solution (12) with the settings blow, but $x, y$ in, respectively, $\left[-5 \times 10^{-6}, 5 \times\right.$ $\left.10^{-6}\right]$ and $\left[-5 \times 10^{-12}, 5 \times 10^{-12}\right]$. The essential property of the fractal structures is the similarity of the figures in different axis scales. Figure 4 demonstrates that the cross-like fractal soliton holds its similarity in different ranges of $x, y$.

\section{Conclusion}

In this paper, we applied an improved mapping method and a variable separation hypothesis to the $(2+1)$-dimensional $\mathrm{vcBK}$ equations and obtained a general variable separation with two arbitrary functions. Based on the general variable separation solution, abundant novel localized excitations, such as oscillating soliton, multidromion soliton, rouge wave, and cross-like fractal structures, have been constructed. The arbitrary functions in the obtained solutions imply that these solutions have rich spatial structures. And it may be helpful in future studies for the intricate nature world. This method can be also extended to the other higher dimensional nonlinear equations. 


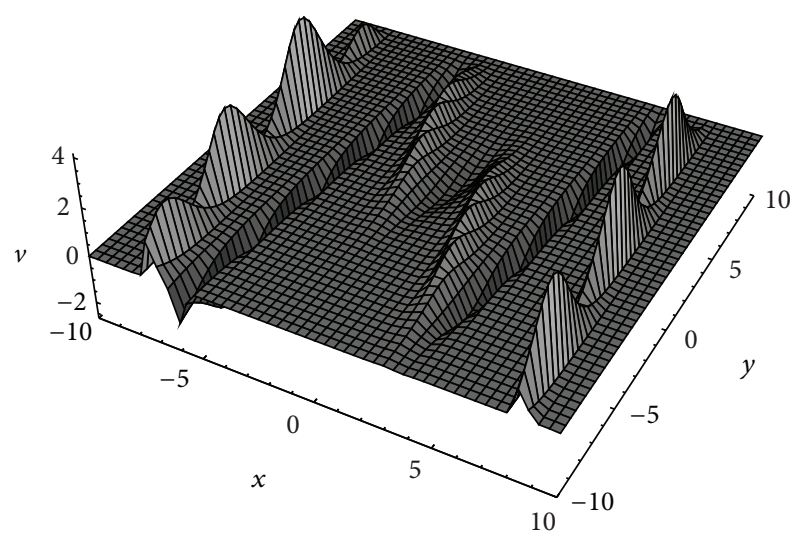

(a)

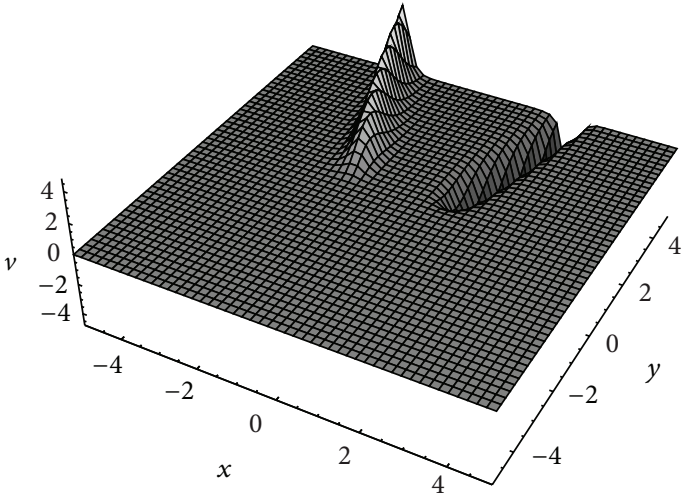

(b)

FIgURe 3: (a) Dromion of periodic oscillation structure $(\delta=-1, t=0)$. (b) Parabola-type kink solitary wave structure $(\delta=-4, t=0)$.

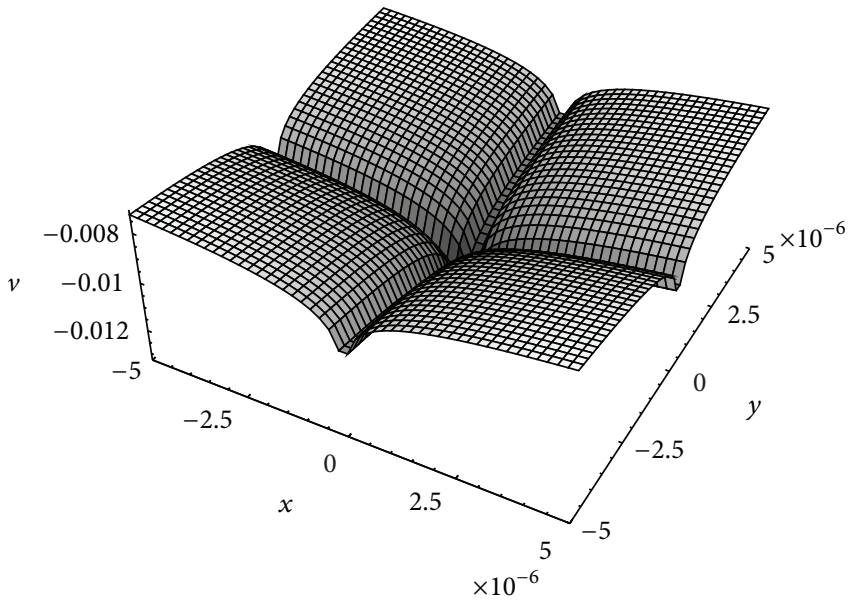

(a)

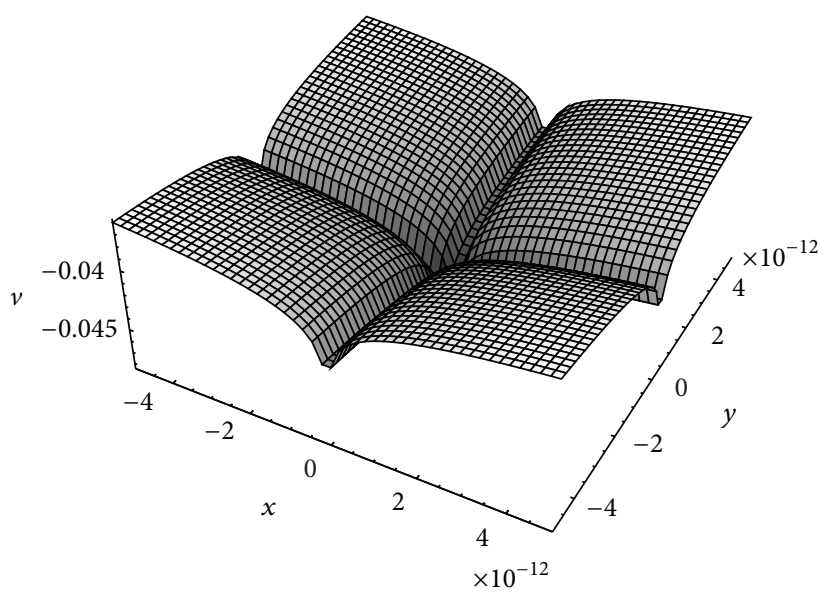

(b)

Figure 4: Cross-like fractal structures $(\delta=-1, t=0)$ : (a) $x \in\left(-5 \times 10^{-6}, 5 \times 10^{-6}\right), y \in\left(-5 \times 10^{-6}, 5 \times 10^{-6}\right)$ and $(\mathrm{b}) x \in\left(-5 \times 10^{-12}, 5 \times 10^{-12}\right)$, $y \in\left(-5 \times 10^{-12}, 5 \times 10^{-12}\right)$.

\section{Conflict of Interests}

The author declares that there is no conflict of interests regarding the publication of this paper.

\section{Acknowledgments}

This paper was supported by Yunnan Province Natural Science Foundation under Grant no. 2013FZ113 and Yunnan Province Educational Department Foundation under Grant no. 2014 Y441.

\section{References}

[1] M. J. Ablowitz and P. A. Clarkson, Solitons, Nonlinear Evolution Equations and Inverse Scattering Transform, vol. 149 of London Mathematical Society Lecture Note Series, Cambridge University Press, Cambridge, UK, 1991.

[2] M. Jimbo and T. Miwa, "Solitons and infinite-dimensional Lie algebras," Publications of the Research Institute for Mathematical Sciences, vol. 19, no. 3, pp. 943-1001, 1983.
[3] Z. Dai, Z. Li, Z. Liu, and D. Li, "Exact cross kink-wave solutions and resonance for the Jimbo-Miwa equation," Physica A, vol. 384, no. 2, pp. 285-290, 2007.

[4] Z. Dai, S. Li, Q. Dai, and J. Huang, "Singular periodic soliton solutions and resonance for the Kadomtsev-Petviashvili equation," Chaos, Solitons \& Fractals, vol. 34, no. 4, pp. 1148-1153, 2007.

[5] Z.-D. Dai, Z.-J. Liu, and D.-L. Li, "Exact periodic solitary-wave solution for KdV equation," Chinese Physics Letters, vol. 25, no. 5, pp. 1531-1533, 2008.

[6] Z. Dai, Z. Li, Z. Liu, and D. Li, "Exact homoclinic wave and soliton solutions for the 2D Ginzburg-Landau equation," Physics Letters A, vol. 372, no. 17, pp. 3010-3014, 2008.

[7] R. Beals, M. Rabelo, and K. Tenenblat, "Bäcklund transformations and inverse scattering and for some pseudospherical surface equations," Studies in Applied Mathematics, vol. 81, pp. 125-151, 1988.

[8] Z. T. Li and Z. D. Dai, "Exact periodic cross kink wave solutions and breather type of two-solitary wave solutions for the $(3+1)$-dimensional potential-YTSF equation," Computers 
\& Mathematics with Applications, vol. 61, no. 8, pp. 1939-1945, 2011.

[9] Z. Dai, S. Lin, H. Fu, and X. Zeng, "Exact three-wave solutions for the KP equation," Applied Mathematics and Computation, vol. 216, no. 5, pp. 1599-1604, 2010.

[10] J.-P. Ying and S.-Y. Lou, "Multilinear variable separation approach in (3+1)-dimensions: the burgers equation," Chinese Physics Letters, vol. 20, no. 9, pp. 1448-1451, 2003.

[11] L. Huang, J. A. Sun, F. Q. Dou, W. Duan, and X. X. Liu, "New variable separation solutions, localized structures and fractals of the (3+1)-dimensional nonlinear Burgers system," Acta Physica Sinica, vol. 56, no. 2, pp. 611-619, 2007.

[12] Z. Li, "New exact homoclinic wave and periodic wave solutions for the Ginzburg-Landau equation," Applied Mathematics and Computation, vol. 217, no. 4, pp. 1549-1554, 2010.

[13] Z. Li and X. Zhang, "New exact kink solutions and periodic form solutions for a generalized Zakharov-Kuznetsov equation with variable coefficients," Communications in Nonlinear Science and Numerical Simulation, vol. 15, no. 11, pp. 3418-3422, 2010.

[14] S. A. El-Wakil and M. A. Abdou, "New exact travelling wave solutions of two nonlinear physical models," Nonlinear Analysis. Theory, Methods \& Applications, vol. 68, no. 2, pp. 235-245, 2008.

[15] C.-L. Zheng, J.-P. Fang, and L.-Q. Chen, "New variable separation excitations of a (2+l)-dimensional broer-kaup-kupershmidt system obtained by an extended mapping approach," Zeitschrift für Naturforschung-Section A, vol. 59, no. 12, pp. 912-918, 2004.

[16] J. F. Zhang, Y. M. Wang, M. L. Wang, and Z. D. Fang, "Exact solutions to the (2+1)-dimensional Broer-Kaup equation with variable coefficients," Journal of Atomic and Molecular Physics, vol. 20, p. 92, 2003.

[17] S. H. Ma, Q. B. Ren, J. P. Fang, and C. L. Zheng, "Special soliton structures and the phenomena of fission and annihilation of solitons for the $(2+1)$-dimensional Borer-Kaup system with variable coefficients," Acta Physica Sinica, vol. 16, pp. 144-149, 2007.

[18] S. Kumar, K. Singh, and R. K. Gupta, "Painlevé analysis, Lie symmetries and exact solutions for (2+1)-dimensional variable coefficients Broer-Kaup equations," Communications in Nonlinear Science and Numerical Simulation, vol. 17, no. 4, pp. 15291541, 2012.

[19] C.-L. Zheng and L.-Q. Chen, "Solitons with fission and fusion behaviors in a variable coefficient Broer-Kaup system," Chaos, Solitons and Fractals, vol. 24, no. 5, pp. 1347-1351, 2005.

[20] T. Geng, W.-R. Shan, X. Lü, K.-J. Cai, C. Zhang, and B. Tian, "New solitary solutions and non-elastic interactions of the (2+1)-dimensional variable-coefficient Broer-Kaup system with symbolic computation," Chaos, Solitons and Fractals, vol. 42, no. 4, pp. 2230-2235, 2009.

[21] B. Li and Y. Ma, "The non-traveling wave solutions and novel fractal soliton for the $(2+1)$-dimensional Broer-Kaup equations with variable coefficients," Communications in Nonlinear Science and Numerical Simulation, vol. 16, no. 1, pp. 144-149, 2011. 


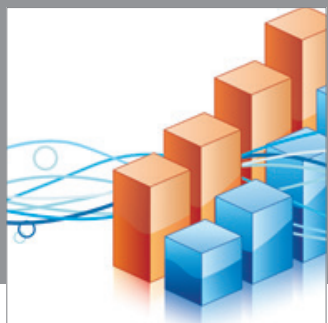

Advances in

Operations Research

mansans

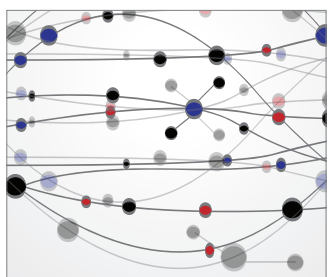

The Scientific World Journal
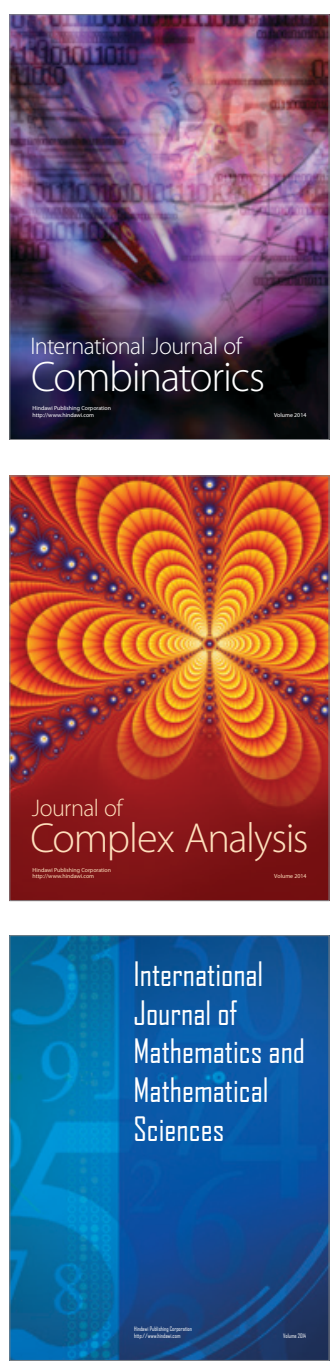
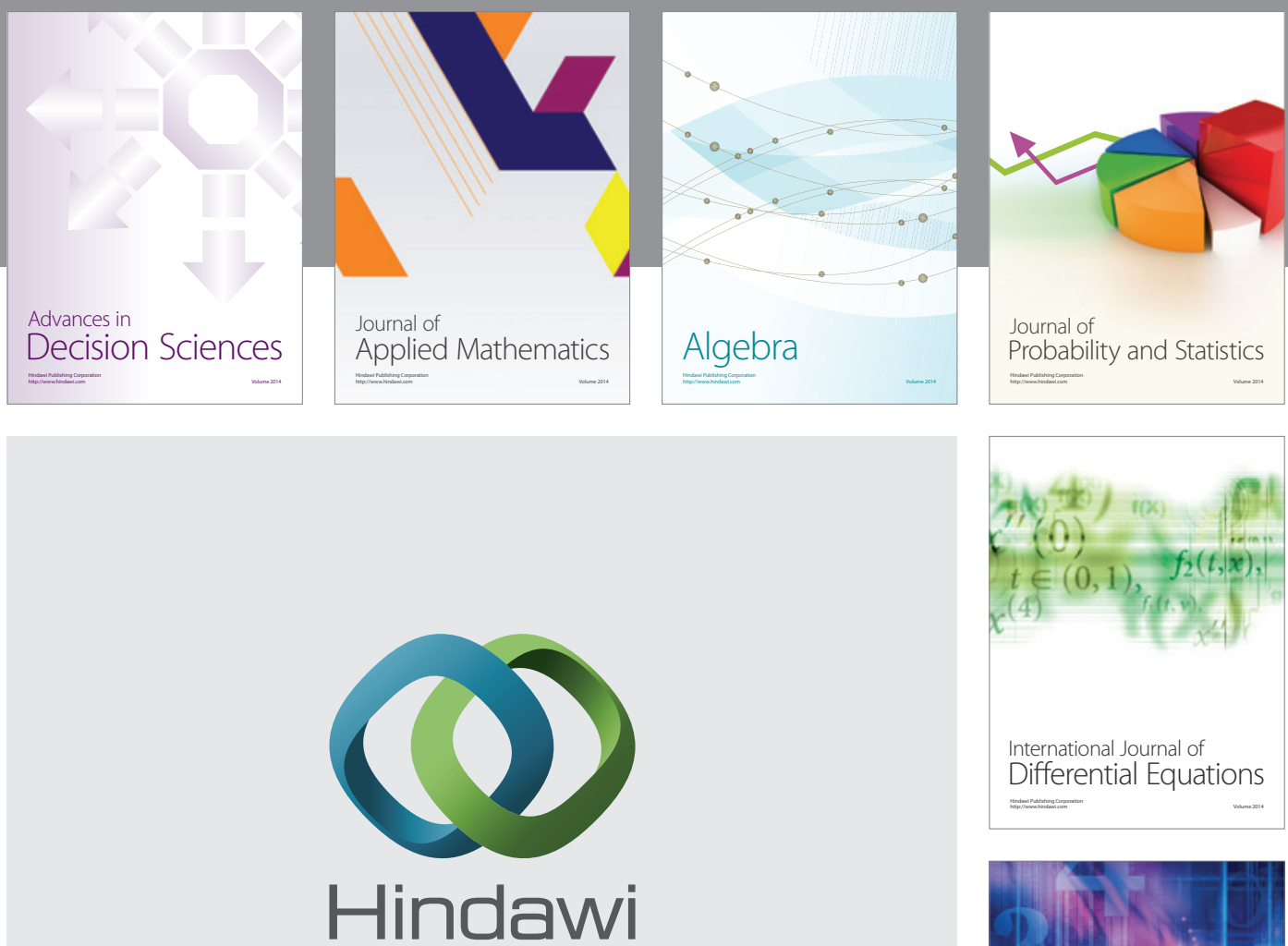

Submit your manuscripts at http://www.hindawi.com
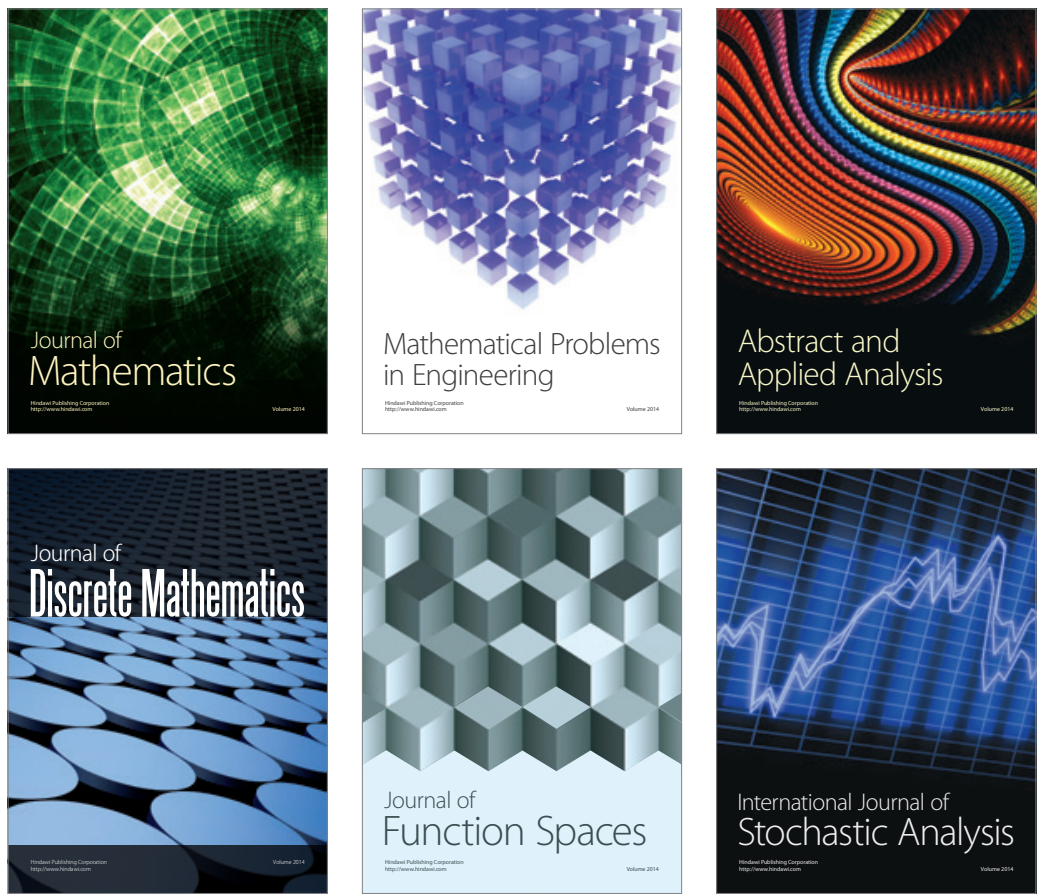

Journal of

Function Spaces

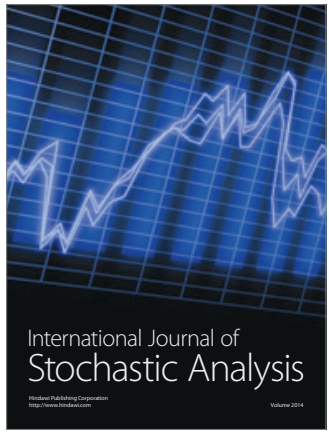

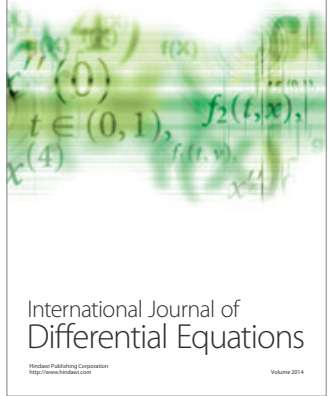
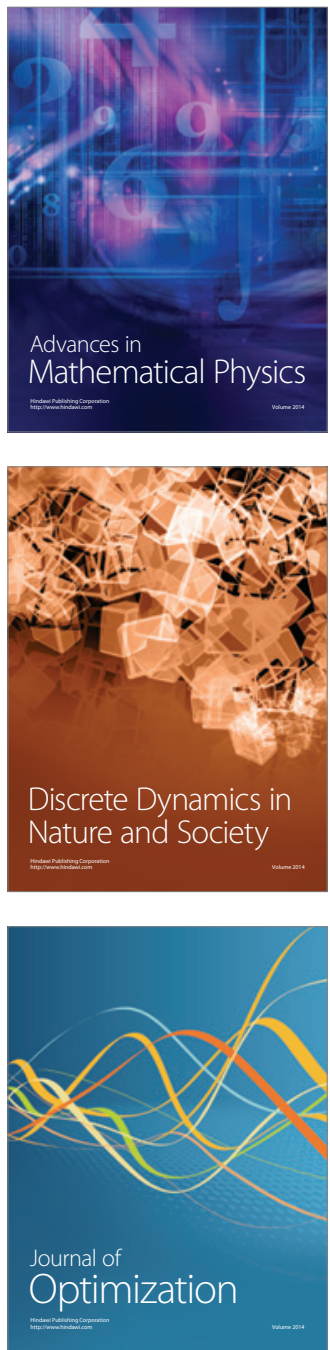